This item was submitted to Loughborough's Research Repository by the author.

Items in Figshare are protected by copyright, with all rights reserved, unless otherwise indicated.

\title{
Applying Object-Oriented Bayesian Networks for smart diagnosis and health monitoring at both component and factory level
}

\section{PLEASE CITE THE PUBLISHED VERSION}

https://link.springer.com/book/10.1007/978-3-319-60045-1

\section{PUBLISHER}

Springer

VERSION

AM (Accepted Manuscript)

\section{PUBLISHER STATEMENT}

This work is made available according to the conditions of the Creative Commons Attribution-NonCommercialNoDerivatives 4.0 International (CC BY-NC-ND 4.0) licence. Full details of this licence are available at: https://creativecommons.org/licenses/by-nc-nd/4.0/

\section{LICENCE}

CC BY-NC-ND 4.0

\section{REPOSITORY RECORD}

Madsen, Anders L., Nicolaj Sondberg-Jeppesen, Mohamed S. Sayed, Michael Peschl, and Niels Lohse. 2019. "Applying Object-oriented Bayesian Networks for Smart Diagnosis and Health Monitoring at Both Component and Factory Level”. figshare. https://hdl.handle.net/2134/24488. 


\title{
Applying Object-Oriented Bayesian Networks for Smart Diagnosis and Health Monitoring at both Component and Factory Level
}

\author{
Anders L. Madsen ${ }^{12}$, Nicolaj Søndberg-Jeppesen ${ }^{1}$, Mohamed S. Sayed ${ }^{3}$, \\ Michael Peschl ${ }^{4}$, and Niels Lohse ${ }^{3}$ \\ 1 HUGIN EXPERT A/S, Aalborg, Denmark \\ 2 Aalborg University, Denmark \\ 3 Loughborough University, United Kingdom \\ 4 Harms \& Wende GmbH \& Co. KG, Hamburg, Germany
}

\begin{abstract}
To support health monitoring and life-long capability management for self-sustaining manufacturing systems, next generation machine components are expected to embed sensory capabilities combined with advanced ICT. The combination of sensory capabilities and the use of Object-Oriented Bayesian Networks (OOBNs) supports self-diagnosis at the component level enabling them to become self-aware and support self-healing production systems. This paper describes the use of a modular component-based modelling approach enabled by the use of OOBNs for health monitoring and root-cause analysis of manufacturing systems using a welding controller produced by Harms \& Wende (HWH) as an example. The model is integrated into the control software of the welding controller and deployed as a SelComp using the SelSus Architecture for diagnosis and predictive maintenance. The SelComp provides diagnosis and condition monitoring capabilities at the component level while the SelSus Architecture provides these capabilities at a wider system level. The results show significant potential of the solution developed.
\end{abstract}

Keywords: OOBNs, Real-World Application, Software Architecture

\section{Introduction}

A Bayesian network (BN) $[11,1-4]$ is a powerful and popular model for probabilistic inference. Its graphical nature makes it well-suited for representing complex problems where the interactions between entities represented as variables are described using conditional probability distributions (CPDs). A Bayesian network is an efficient knowledge integration tool enabling information from different sources such as mathematical formulas, historical data and domain expert knowledge to be combined into a single model. As such they have been used in a wide range of domains for managing uncertainty.

We describe the application of OOBNs for health monitoring and root-cause analysis of manufacturing systems using a welding controller produced by Harms \& Wende $(\mathrm{HWH})$ as an example. HWH serves tens of thousands of welding 
equipments worldwide. Today, three service technicians are permanently available on phone hotline for solving customer problems. Obviously, one can reduce the load on this personnel by implementing a root-cause analysis solution at the customer service center combined with health monitoring at component level. Several benefits are expected from the successful implementation of such a tool. The average service technician training time is now six months. By implementing and using a service analysis solution this training time can be reduced to two weeks. HWH has about 20 requests per week or about 1000 requests in a year. HWH expects that by using an analytical software solution the average service request processing time will be reduced from 1 hour to less than $40 \mathrm{~min}$. This in turn should result in a reduction of load on each employee, improvement of service quality by eliminating human errors, and 333 hours of savings annually.

The HWH OOBN model for component-based diagnostic has been encapsulated as a SelComp in the SelSus software architecture to enable system-level diagnostic capabilities [14]. We describe the integration and present the results of a performance evaluation of different levels of integration (direct, local network and wider network). There is a fair amount of related work on the use of OOBNs for diagnosis of industrial equipment including $[8,15,6,12]$.

\section{Preliminaries and Notation}

A BN $[11,1-4]$ consists of two main components. The first component is a graphical structure specifying dependence and independence relations between the random variables of the model and the second component is a set of CPDs specifying the strengths of the dependence relations. More precisely, a BN is a

pair $\langle\mathcal{G}, \mathcal{P}\rangle$, where $\mathcal{G}=(\mathbf{V}, \mathbf{E})$ is an acyclic, directed graph (DAG) over a set of random variables $\mathcal{X} \sim \mathbf{V}$ with directed edges $\mathbf{E}$ that represent probabilistic relationships between variables $\mathcal{X}$ and $\mathcal{P}$ is the set of CPDs. This means that a $\mathrm{BN}$ is a decomposition of a joint probability distributions as follows $P(\mathcal{X})=P\left(X_{1}, \ldots, X_{n}\right)=\prod_{X_{i} \in \mathcal{X}} P\left(X_{i} \mid p a\left(X_{i}\right)\right)$. A BN supports the calculation of the posterior probability $P\left(X_{i} \mid \epsilon\right)$ where $\epsilon$ is the observed evidence and $X_{i}$ is any non-observed variable.

An OOBN can been seen as a Bayesian network augmented with network classes, class instances and an associated notion of interface and private variables $[5,10,3]$. A class instance is the instantiation of a network class representing a sub-network within another network class. An OOBN can be used to represent a problem domain with repetitive structures more compactly and supports efficient model reuse as well as distributed knowledge elicitation. The variables $\mathcal{X}(C)$ of network class $C$ are divided into disjoint subsets of input $\mathcal{I}$, output $\mathcal{O}$ and hidden/private $\mathcal{H}$ variables such that $\mathcal{X}(C)=\mathcal{I} \cup \mathcal{O} \cup \mathcal{H}$ where the interface variables $\mathcal{I} \cup \mathcal{O}$ are used to link nested class instances.

The OOBN for the HWH welding controller has been developed following a six steps methodology [8] that has proven to be efficient and effective in practice. The six steps of the model development cycle are begin, design, implement,

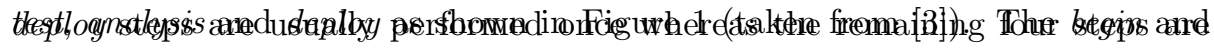




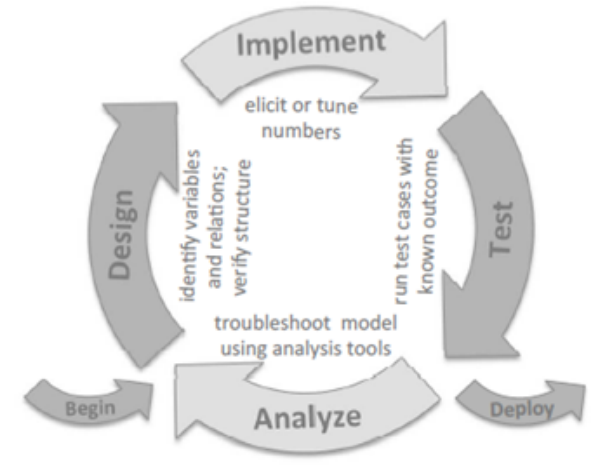

Fig. 1. Model development cycle [3].

iterated until the model performance requirements are satisfied or until further improvements are not possible or too costly.

\section{HWH Welding Controller}

The objective of this work has been to realize functionality that allows fast and efficient root-cause analysis of failures and health monitoring of a welding system in the field. Because of the huge amount of components of such a system, e.g., welding gun, transformers, cables, cooling systems and welding control including its sub-components, and their complex interrelationships, root-cause analysis nowadays is a manual task which consumes a lot of time. Often, the root causes can only be identified after several iterations including phone conversations with the customer, logging data analysis or even time-consuming in situ analysis.

Here, we consider the HWH welding controller Genius MFI in combination with the control software XPegasus. The Genius MFI has two separated parts of electronics: the power electronic and the cards electronic. The power electronic provides high power output for performing the welding. The cards electronic is used to control the power electronic and to connect the control with peripheral hardware and software components. The welding controller can be parametrized by the XPegasus PC software. XPegasus also supports documentation and analysis of welding data. The welding controller and XPegasus are connected via TCP/IP over Ethernet. Figure 2(a) shows the XPegasus interface.

XPegasus has a component-oriented software architecture including a huge amount of components, e.g., components for data analysis, quality inspection and client-server connectivity already exist. The objective is to provide the analytical software solution both to HWH service personnel as well as to the customers of HWH. The integration of diagnosis capabilities into the equipment provides the following benefits: 1) reaction time in case of failures reduced from up to 12 hours to maximum 1 hour (due to different time zones), 2) many problems can be solved locally without contacting HWH customer service center. This will result 


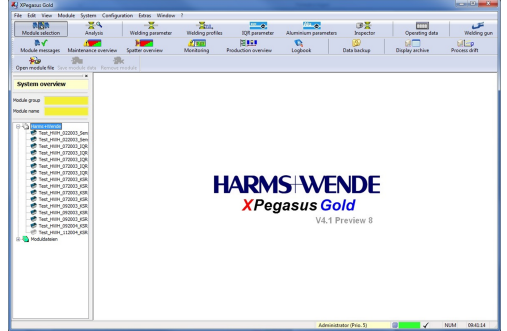

(a) XPegasus.

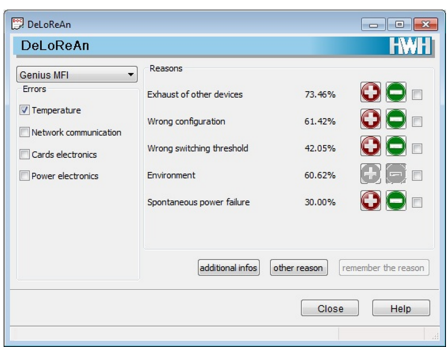

(b) Diagnosis Capabilities

Fig. 2. The XPegasus start screen and diagnosis capabilities.

in an additional reduction of the load on service personnel, and 3) the embedded analytical software will support early identification of potential problems/failures and provide warnings to avoid them. This results in a substantial qualitative improvement of the equipment itself. Considering all the factors listed above, the use of OOBNs is expected to substantially improve Overall Equipment Efficiency (OEE) and reduce investments.

\section{OOBN for HWH Welding Controller}

This section describes the development of the component-level diagnosis model for the welding controller produced by HWH using the method of [8]. The results of the six steps of the method are described next. The model was developed during two physical modelling workshops, numerous web meetings and email exchanges. The process was launched with a physical workshop.

\subsection{Begin: Model Choice}

The HWH diagnosis model has been developed as an OOBN since it is to be used for root-cause analysis at the component level as well as to be integrated into a larger system-wide model for root-cause analysis at a higher level of abstraction, i.e., shop-floor or even factory level. The model is developed using HUGIN software $[9]^{5}$. An OOBN has the advantage of supporting model reuse and can be extended into a dynamic model to support predictive maintenance.

\subsection{Design: Structure}

As part of the design step, domain experts from HWH and knowledge engineers identified possible root causes, mediating variables and a set of possible observables (e.g., an observation by the operator or a sensor reading). Figure 3 shows the top level network class that was the output of the Design-step. The OOBN

\footnotetext{
${ }^{5}$ http://www.hugin.com
} 
reflects root causes, possible observations and whether a problem has been reported or not. The model contains four instance nodes that represent instances of embedded components (white boxes with rounded corners). Figure 4 shows

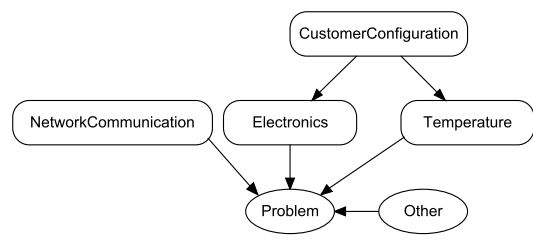

Fig. 3. The top level class of the Welding Controller model.

the network class representing the Power Electronics of the welding controller. This network class has one instance of the network class representing the parameter settings. The Power Electronics class is instantiated in the Electronics class (not shown) instantiated in the top level class. The OOBN has a total of eight

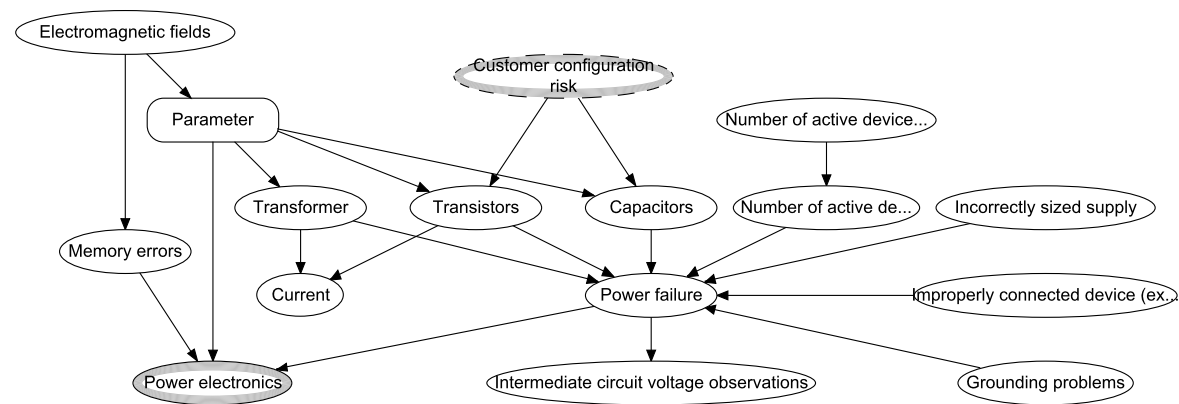

Fig. 4. The Power Electronics network class.

network classes and four layers in the class component tree (number of nested classes). The unfolded model has 24 Boolean root causes, 8 observations, i.e., sensor readings and operator input and a total of 48 variables with a total CPD size of 623 . Notice that some root causes have parents in the structure and that the number of observations is relatively low.

\subsection{Implement: Quantification}

In order to assess the root cause probabilities, domain experts were asked to estimate the prior probability of a problem and provide a ranking of the root 
causes taking conditional variables into consideration. Each root cause was assigned a prior probability proportional to its ranking. Since each root cause is represented as a Boolean variable the conditional probability distributions for the problem defining variables are mainly specified as a logical disjunction of the parent nodes. In some cases, a small leak probability is added to represent that some unlikely or unknown failures are not represented in the model or that a problem is not observed yet even though a root cause is present. Distributions for sensor readings and operator observations have been assessed by experts.

\subsection{Test and Analysis}

The model was first evaluated qualitatively by domain experts using a web interface and the XPegasus software. Secondly, it was evaluated quantitatively by means of a synthetic data set that was produced by random sampling from the model. For each possible root cause, a set of observations was sampled from the model (given the root cause being set to a failure state and all other causes set to non-failure). Next, the sampled observations and a problem being observed were entered as evidence. The probability of each root cause was retrieved. Ideally, the original root cause would have the highest probability in every case. This evaluation showed that in six cases the true root cause had the highest probability while in 16 out of 21 cases the true root cause was between the five root causes with highest probability.

Due to a low number of sensor readings and operator observations, the model is not able to distinguish between certain root causes. For instance, the model contains no evidence variables to distinguish root cause variables Grounding problems and Incorrectly sized supply as they share one common sensor reading.

\subsection{Deploy}

The model has been integrated directly into the control software of the welding controller in order to support diagnosis at the component level as well as deployed inside the SelSus architecture. This is described in more detail in the next section. In addition, a special-purpose web interface [7] for the welding controller model was developed to support the Test-step of the development process ${ }^{6}$. This has served as an important tool in the Test and Analysis-step as the domain experts have been able to interact with the model at their own convenience.

\section{The SelSus Architecture}

The SelSus architecture is primarily based on the concept of independent smart automation components that can be integrated in a bottom-up fashion into a wider automation system [14]. The underlying intention is to enable the providers of automation components, such as HWH, to offer more encapsulated diagnostic and monitoring functionality as part of their device offering while enabling faster integration and reconfiguration of devices into automation systems.

\footnotetext{
${ }^{6}$ http://selsus.hugin.com
} 


\subsection{The SelComp Concept}

The automation components in SelSus are encapsulated using the concept of a SelComp. The aim is to encapsulate the automation component with its own control and sensory capabilities including some embedded data processing, diagnostic and prognostic capabilities. A SelSus system is an integration of a number of SelComps with the added system-level functionalities on the SelSus cloud. This enables diagnostic and prognostic reasoning to be conducted on two levels: locally on the SelComp level where the only issues within the scope of the SelComp itself are possible to be detected and analysed; and System-level reasoning where various SelComps make some or all of their observations available for system-level reasoning. This enables system-level visibility in capturing and analysing the state of the system as a whole covering the various influences between individual SelComps. Figure 5 shows an overview of the generic internal architecture of the SelComp and how it relates to the system-level functionalities within SelSus, while Figure 2(b) shows how the diagnosis capabilities offered by the model at the SelComp level has been made available to the user of XPegasus. The OOBN approach enables component-based encapsulated modelling at the

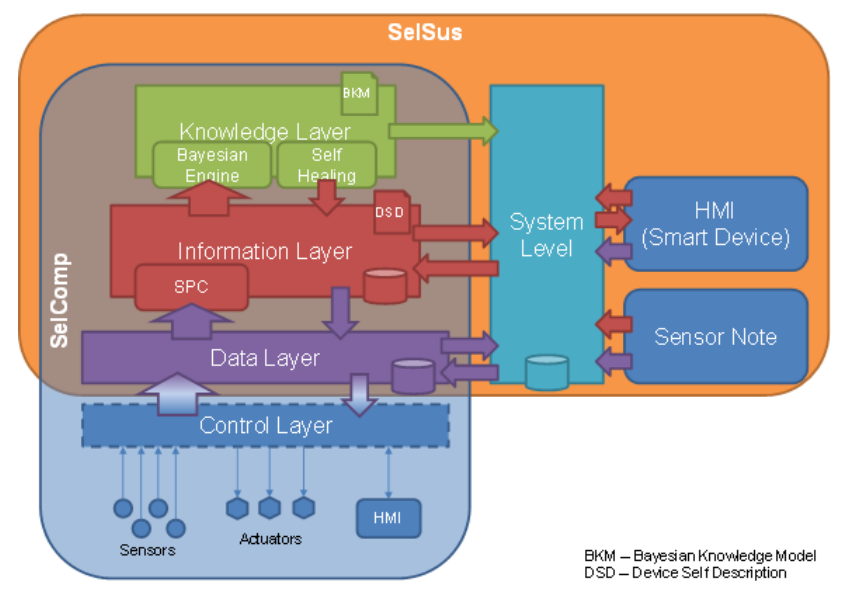

Fig. 5. The SelComp internal architecture concept.

SelComp level, while enabling the individual SelComp models to be integrated into a wider system-level model that can be used at system level.

\subsection{Wider System Level Diagnosis}

Following the notions of component-based modelling the aim here is to enable modular, component-based OOBN models to be constructed on the SelComp level while accommodating for the possibility of integrating these local models 
into a wider system-level model that covers the overall system. The component models should ideally be self-sufficient and only require information from the available information to the SelComp.

The objective is to enable independent SelComps to operate normally even when they are not connected to an overall SelSus system. This can only be true if the diagnostic and prognostic models along with the reasoning process are entirely self-contained within each individual SelComp and do not depend in their operation on external resources, either in the form of observations or reasoning and computation. The system-level models will be a collection of the constituent component-models. In the context of manufacturing systems, individual component models will typically be connected together through the means of process quality characteristics in order to form system-level diagnostic models.

In the use-case we consider, the SelComp model presented could be linked to other SelComp models representing other components in an integrated system in which the HWH welding controller is deployed. This is ongoing work.

\subsection{The HWH diagnosis model as a SelSus Cloud Service}

In the SelSus architecture, SelComps have the ability to transmit data to and receive data from the SelSus Cloud. The SelSus Cloud is a collection of Software Services from which a SelComp can request (stored) sensor data or it can communicate with, for instance, another Service that is exposing the HUGIN functionality. This latter web service can be equipped with the welding controller model, when requested - thus allowing a SelComp to perform diagnosis and health monitoring on the HWH welding controller. Specifically, this web service communicates using a REST based API where requests can be bundled in JSON objects to minimize the communication overhead.

\subsection{Experimental Analysis}

This section reports on an experimental analysis of the performance of different levels of integration of the HWH model into the SelSus architecture. The tightest level of integration has been achieved by integrating the model directly into the XPegasus software. In addition, the model has been deployed using a web service inside the SelSus Cloud having the control software and web service running on the same machine as well as having the control software and web service running on machines located far apart (more than $1000 \mathrm{~km}$ ). In the experiment, one state for each possible observation was propagated and this process was repeated 1000 times producing 8000 propagations. Table 1 shows the time performance results for different configurations. It is clear from the table that direct integration into the control software using save-to-memory ${ }^{7}$ is by far the most time efficient solution. Using this approach it is possible to perform thousands of propagations in the model each second using the test computer, which is a standard PC.

\footnotetext{
${ }_{7}$ save-to-memory is an optimisation option in HUGIN software trading time for space.
} 
Table 1. Average time cost of one belief update across three different setups.

\begin{tabular}{|l|r|r|}
\hline Configuration & Total time $(\mathrm{ms})$ & Average time $(\mathrm{ms})$ \\
\hline Direct integration & 1,508 & 0.189 \\
Direct integration (w/save-to-memory) & 778 & 0.097 \\
Localhost deployment & 10,263 & 1.283 \\
Network deployment & 382,785 & 47.848 \\
\hline
\end{tabular}

\section{Discussion and Conclusion}

The use of Bayesian networks for diagnosis at the component level has several benefits to HWH. For HWH, in general, this will result in a substantial qualitative improvement of welding equipment and reduction of costs related to service. A qualitative improvement implies that equipment will become more intelligent, reliable, stable and predictive and human-friendly. In addition, these new capabilities provide competitive advantages.

For the HWH customer service center, the new software will reduce the load related to service problem analysis. It will also improve quality of service, reduce training times for new personnel and reduce requirements to their expertise.

For HWH customers, the usage of embedded analytical software provides several advantages. The welding equipment will become more intelligent with self-awareness and self-diagnosis capabilities. Self-awareness helps in easy integration and configuration into the system while self-diagnosis will help in predicting and preventing failures or finding solutions in case of problems/failures. For the customer this means a substantial simplification of equipment service, improvement of production line efficiency and stability by reducing down-times and failures. All this results in a significant reduction of costs.

The results of the experimental analysis clearly demonstrate that inference is highly efficient using the tightest level of integration and that communication overhead adds significantly to the time cost of the inference process. Even with the deployment of the web service and control software on different machines located far apart, the cost of inference should not be problematic in relation to providing support on root-cause analysis over the phone.

Future work includes development of a system-wide model for diagnosis at line or even factory level combining the HWH welding controller model with models for other components on the line or factory level as well as collecting operational data for parameter estimation in batch and considering algorithms for on-line adjustment of parameters using operational data. Although the modelling methodology is primarily driven by domain expert knowledge, current work focuses on enabling the derivation of Bayesian network models for diagnosis from existing engineering design information such as FMEA data [13].

\section{Acknowledgments}

This work is part of the project "Health Monitoring and Life-Long Capability Management for SELf-SUStaining Manufacturing Systems (SelSus)" which is 
funded by the Commission of the European Communities under the 7th Framework Programme, Grant agreement no: 609382.

\section{References}

1. R.G. Cowell, A.P. Dawid, S.L. Lauritzen, and D.J. Spiegelhalter. Probabilistic Networks and Expert Systems. Springer, 1999.

2. F. V. Jensen and T. D. Nielsen. Bayesian Networks and Decision Graphs. Springer, 2nd edition, 2007.

3. U. B. Kjærulff and A. L. Madsen. Bayesian Networks and Influence Diagrams: A Guide to Construction and Analysis. Springer, 2nd edition, 2013.

4. D. Koller and N. Friedman. Probabilistic Graphical Models - Principles and Techniques. MITPress, 2009.

5. Daphne Koller and Avi Pfeffer. Object-oriented Bayesian networks. In Proc. of $U A I$, pages 302-313, 1997.

6. A. Lorenzoni and M. Kempf. Degradation processes modelled with dynamic bayesian networks. In Proceedings of the 2015 IEEE International Conference on Industrial Informatics (INDIN), page 6, 2015.

7. A. L. Madsen, M. Karlsen, G. B. Barker, A. B. Garcia, J. Hoorfar, F. Jensen, and H. Vigre. A Software Package for Web Deployment of Probabilistic Graphical Models. In Proc. of SCAI, pages 175-184, 2013.

8. A. L. Madsen, N. Søndberg-Jeppesen, N. Lohse, and M. S. Sayed. A methodology for developing local smart diagnostic models using expert knowledge. In Proceedings of the 2015 IEEE International Conference on Industrial Informatics (INDIN), page 6, 2015.

9. A.L. Madsen, F. Jensen, U.B. Kjærulff, and M. Lang. HUGIN - The Tool for Bayesian Networks and Influence Diagrams. International Journal on Artificial Intelligence Tools 14, 3:507-543, 2005.

10. M. Neil, N. Fenton, and L. M. Nielsen. Building large-scale Bayesian networks. The Knowledge Engineering Review, 15(3):257-284, 2000.

11. J. Pearl. Probabilistic Reasoning in Intelligent Systems: Networks of Plausible Inference. Series in Representation and Reasoning. Morgan Kaufmann Publishers, San Mateo, CA, 1988.

12. M. S. Sayed and N. Lohse. Distributed bayesian diagnosis for modular assembly systemsa case study. Journal of Manufacturing Systems, 32(3):480 - 488, 2013.

13. M. S. Sayed and N. Lohse. Ontology-driven generation of Bayesian diagnostic models for assembly systems. International Journal of Advanced Manufacturing Technology, 74(5-8):1033-1052, 2014.

14. M. S. Sayed, N. Lohse, N. Søndberg-Jeppesen, and A. L. Madsen. Selsus: Towards a reference architecture for diagnostics and predictive maintenance using smart manufacturing devices. In Proceedings of the 2015 IEEE International Conference on Industrial Informatics (INDIN), page 6, 2015.

15. G. Weidl, A. L. Madsen, and S. Israelson. Applications of object-oriented bayesian networks for condition monitoring, root cause analysis and decision support on operation of complex continuous processes. Computers and Chemical Engineering, 29:19962009, 2005. 